\title{
EDITORIAL
}

\section{Revisiting Plant Fats and Health in the Caribbean}

\author{
FJ Henry
}

Three popular plant foods have traditionally received negative health ratings in the Caribbean. These are the products of coconut (Cocos nucifera), avocado (Persea americana) and ackee (Blighia sapida) mainly because of their well-established fat content. Over the years, research has shown that the purported health risks are unfounded simply because all fats are not equal. The most recent finding (1), reported in this issue of the Journal, suggests that ackee is not harmful and may even be beneficial to health if eaten in appropriate quantities. Such new findings have profound significance beyond public health. Previous false and misleading information about the nutrient content of local foods has caused long-term and almost irreparable damage to consumption and the export market for indigenous Caribbean food crops.

The concern is created because fat is the most concentrated source of energy in the diet and obesity is the most important underlying cause of death in the region. Further, specific fatty acids have been linked to cancers and other chronic diseases. But the ingestion of fat has to be placed in the context of use, quantity and type. For example, being concentrated in energy is often good for persons who can only eat small amounts and are unable to meet their energy needs.

Moreover, fats also have many important roles in the body, for example, they carry vital, soluble substances such as vitamins $\mathrm{A}, \mathrm{D}, \mathrm{E}$ and $\mathrm{K}$. But the focus on fat in recent decades has been on the excess fat intake which is stored in various fatty (adipose) tissues. This accumulation of fatty tissues is responsible for up to three-quarters of the Caribbean adult population being overweight or obese. It is important to note that sugar is also a major contributor to the obesity tsunami. Irrespective of origin, the consequences of these diet-related excesses are obesity, heart disease, diabetes, hypertension, stroke and some cancers - all of which lead to disabilities and early death and also cause major financial burden to individual families and the national health system.

In general, the recommendation for good health is to consume predominantly plant rather than animal foods in

From: College of Health Sciences, University of Technology, Kingston, Jamaica.

Correspondence: Professor FJ Henry, Public Health Nutrition, College of Health Sciences, University of Technology, 237 Old Hope Road, Kingston 6, Jamaica. E-mail: fitzroy_henry22@yahoo.com accordance with national dietary guidelines. While animal foods usually have much more fat than plant foods, it is noted that some plant foods contain substantial amounts of fat - particularly avocado, coconut oil and ackee. But the roles and functions of fatty acids differ and hence result in both negative and positive health consequences. It is therefore critical to revisit these plant fats in relation to their respective compositions and the risks or benefits associated with them.

Coconut oil is a saturated fat, but unlike other oils and fats, about $40-50 \%$ of the fatty acid in coconut oil is lauric acid. Lauric acid offers unique properties in food use as well as non-food uses such as the making of natural soaps, other health products and in the cosmetics industry. In food use, there are properties related to its antiviral, antibacterial, and antiprotozoal functions. The combination of lauric, caprylic, oleic and capric fatty acids in coconut oil is a nutritional asset because these medium and short-chain fatty acids are useful in the treatment of certain digestive diseases. Natural coconut oil in the diet leads to a normalization of body lipids because of the presence of lauric acid which, along with other mediumchain fatty acids, does not adversely affect the high-density lipoprotein (HDL)/low-density lipoprotein (LDL) cholesterol ratio or contribute to heart disease, but can actually promote health. Additionally, medium-chain fatty acids are short enough to be absorbed directly into the portal blood and because they bypass the lymph, they can be in the bloodstream in about twenty minutes after ingestion for oxidization in the liver. Avocado contains mainly unsaturated fats with oleic acid contributing $50-80 \%$ of lipids, depending on variety. The saturated fat content is about $14 \%$ (palmitic acid). In addition, avocado is sodium free and a good source of potassium and fibre. It is also a valuable antioxidant through its vitamin A, C and E content. Further, avocado lowers blood cholesterol, is rich in folic acid and vitamin $\mathrm{K}$ and as such can be a healthy option if used appropriately.

Ackee is an unsaturated fat, and has additional health benefits through its high protein content, being a good source of vitamins B and C, zinc, calcium and fibre. But it is the fatty acid composition that is of great significance to health outcomes. It was previously found that $51-58 \%$ of lipids in ackee contain mainly linoleic and stearic fatty acids. The new finding (1), which resulted from a more robust methodology, shows minor to undetectable levels of linoleic acid and 13\% stearic acid. Importantly, however, the dominant lipid (55\%) 
is oleic acid. Although the negative health consequences of linoleic and stearic acid are not compelling $(2,3)$, oleic acid, in contrast, has a very positive effect on HDL/LDL cholesterol ratio and reduces the risk of chronic diseases, breast cancer in particular $(4,5)$. If this new finding (1) holds true in subsequent studies, it can dramatically change the perception and use of this tasty and commonly used ackee dish in Jamaica and other countries. No longer will the fear of cancer risk be held; in fact, the judicious consumption of this food can be positively marketed locally and abroad.

Plant fats have erroneously received negative ratings over the years. Because of the persistence of researchers, however, these plant fats are slowly taking their rightful place in the culinary and non-food sectors. Ultimately, it must be recognized that ackee, coconut oil and avocado have a high fat content and the excess energy will be stored as fat which is a major contributor to obesity and non-communicable diseases, the most important health problems in the Caribbean. But there is no reason to avoid these low-cost, commonly available and popular foods. These plant fats should be freely used as part of the national dietary guidelines. This will increase the consumption of affordable delicious meals and ensure healthy outcomes.

\section{REFERENCES}

1. Goldson A, Bremmer D, Nelson K, Minott DA. Fat profile of Jamaican ackees, oleic acid content and possible health implications. West Indian Med J 2014; 63: 9-12.

2. Zock PL, Katan MB. Linoleic acid intake and cancer risk: a review and meta-analysis. Am J Clin Nutr 1998; 68: 142-153.

3. Hunter JE, Zhang J, Kris-Etherton PM. Cardiovascular disease risk of dietary stearic acid compared with trans, other saturated and unsaturated fatty acids: a systematic review. Am J Clin Nutr 2010; 91: 46-63.

4. Sales-Campos H, Souza PR, Peghini BC, da silva JS, Cardoso CR. An overview of the modulatory effects of oleic acid in health and disease. Mimi Rev Med Chem 2013; 13: 201-10.

5. Win TD. Oleic acid - the anti-breast cancer component in olive oil. AU J T 2005; 9: 75-8. 